\title{
Computer Independent Data Transfer Device
}

\author{
https://doi.org/10.3991/ijes.v5i2.7038 \\ Darshana Rarath \\ Dr. D Y Patil School of Engineering and Technology, Pune, Maharashtra, India \\ darshana2703@gmail.com \\ Mayank Sharma \\ Dr. D Y Patil School of Engineering and Technology, Pune, Maharashtra, India \\ mayank310795@gmail.com \\ Akshay Mane \\ Dr. D Y Patil School of Engineering and Technology, Pune, Maharashtra, India \\ maneakshayc203@gmail.com \\ Pooja Dabral \\ Dr. D Y Patil School of Engineering and Technology, Pune, Maharashtra, India \\ poojadabral17@gmail.com \\ Roshani Ade \\ Dr. D Y Patil School of Engineering and Technology, Pune, Maharashtra, India \\ rosh513@gmail.com
}

\begin{abstract}
In today's era, transferring data among distinct storage devices has become one of the tasks which are done most frequently. In order to make data and information omnipresent, it needs to be shared anywhere and anytime. However the reliance of user on a PC or laptop for the same is not efficient. This paper is about the innovative way to overcome this restriction. This paper discusses the development of a portable device with the use of wired and wireless communication applications to share data and information among distinct storage devices without relying on a PC or a laptop. The proposed device is compact, comprises of a touch screen, power source and is capable of transferring all types of files. Hence, it eliminates the dependence on a PC or a laptop for transferring data.
\end{abstract}

Keywords-ARM, Bluetooth, Wi-Fi, USB

\section{Introduction}

Nowadays, data and information is stored in distinct storage devices (Pen drives, External hard disks, MP3 players, Phone Memory etc.). However, the presence of a PC or a laptop is very necessary to share data among them. This fact restricts the user to share the data in the absence of a PC. Aim of the proposed system is to allow the user to transfer all types of files in all possible ways without sacrificing mobility. For 
this, Raspberry Pi has been used as a development board which comes with $1.2 \mathrm{GHz}$ 64 bit quad core processor, ARMv8 CPU, 802.11n Wireless LAN, Bluetooth 4.1 for wireless data transfer. It also has 1 GB RAM which gives the device adequate amount of processing power, 4 USB ports which can be used for wired data transfer among USB storage devices (such as Pen Drive to External Hard disk), 40 GPIO pins which can be used to mount a TFT LCD screen.

Rest of the paper has been structured as follows:

Section 2 elaborates the "Literature survey", which contains the description of all the papers which were referred to. Section 3 describes the "Proposed system". It describes the technical support for the system. Section 4 describes the detailed working which contains the algorithm used. Section 5 contains the results obtained after testing. It also shows the comparison of performance of the system with PC. Section 6 contains conclusion. Section 7 contains the future scope and section 8 contains all the references.

\section{$2 \quad$ Literature survey}

Several devices have been developed to overcome the reliance of a user on a PC for transferring data among distinct storage devices. One of the recently developed similar systems had used USB host controller (VNCIL) along with microcontroller ARMLPC 2138 [1]. This system possessed the ability to transfer various types of files (.avi, .docx, .jpg, .pdf, .pptx) among several storage devices in a wired manner. The device also included a separate touch screen board and a 16 X 4 LCD screen to exhibit the files as well as the control options. Along with wired data transfer, it would be a lot more convenient for the users if this device [1] could share the files in a wireless manner. Since nowadays, the use of wireless communication (such as Bluetooth and Wi-Fi) is prevailing; several devices were developed to allow data transfer in a wireless manner. One of these devices [2] is based on the emerging application, the wireless memory access through Bluetooth enabled DTE, which has achieved wireless data transfer through Bluetooth, which enables data to be transferred between a USB storage device and a Bluetooth compatible device. The presence of a Graphical User Interface makes it a lot easier for a user to use a device. One of the devices [3] provided a Graphical User Interface to make it easier for the user to access the content of the storage devices. It allowed the user to choose a specific file from the mass storage device connected to the port and transfer it to the other storage device using the options like copy, explore, list which were displayed on the touch screen. It is very crucial to have an efficient, reliable and fast communication between the user interface and the USB communication module. To achieve this, a file system controller has been used in one the systems [4]. It is responsible for all the file management functions and FAT file system access. It acts as a mediator between the display and the USB module. Whenever a mobile device runs out of storage space, it becomes very necessary to transfer its files to an external storage device which restricts the mobility as it becomes necessary to connect the mobile device to an external storage device using a cable. To overcome this restriction, the system [5] has developed supporting 
tools that allow users to transfer the files between mobile devices and external storage devices through internet. One of the other systems has used the LPC2387 microprocessor to realize the design of USB host in the non-PC application situation [6].

\section{$3 \quad$ Proposed system}

The proposed system facilitates data transfer in a wired, as well as a wireless manner. For ex. transferring data between two pen drives connected to the host controller or between a pen drive and a smart phone where the smart phone is connected to the host controller through Wi-Fi/Bluetooth.

For efficient implementation of the aforementioned features, several components and technologies have been used, which are:

1. Raspberry Pi.

2. ARM Processor.

3. USB

4. Bluetooth Technology.

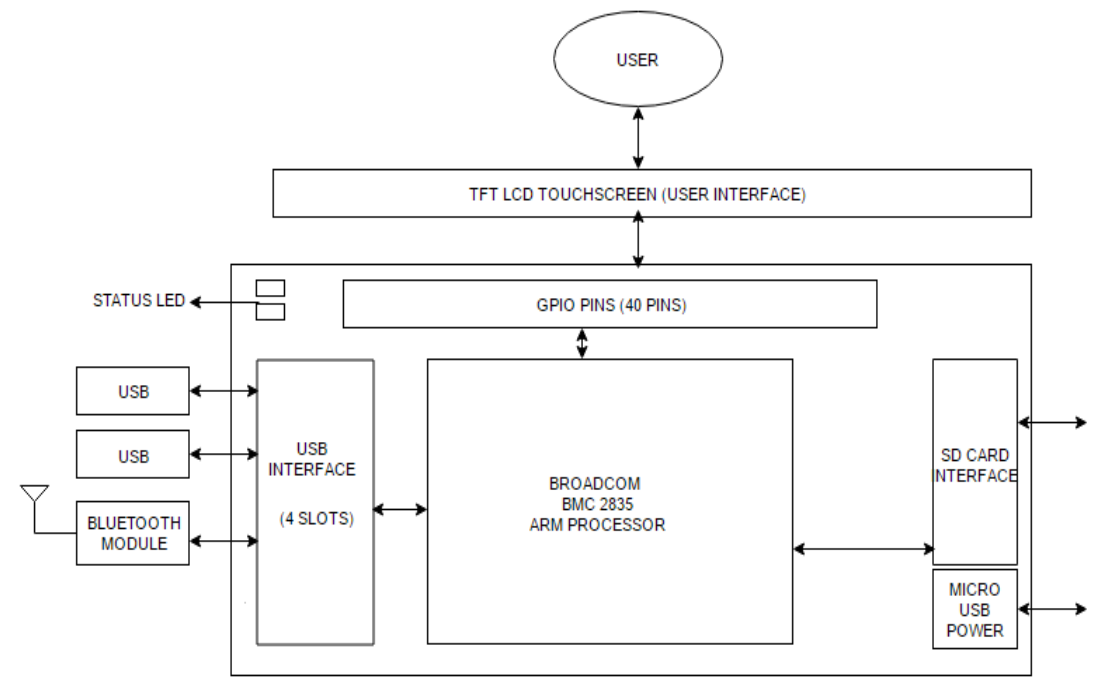

Fig. 1. System Architecture

\subsection{Raspberry Pi}

As mentioned previously, in the proposed system, Raspberry Pi has been used as a development board. It comes with a Broadcom BCM2835 SOC (system on chip) which includes ARM processor. It also includes 4 USB ports which are extremely vital for the system as they give the users options to join new modules to the device. The USB controller contains high speed serial communication ports which provide a baud rate of $12 \mathrm{MBits} / \mathrm{sec}[7]$. 
In order to make the device more user friendly, a Simple User Interface has been provided. For this, the system uses a Thin Film Transistor (TFT) screen [7]. All the content of USB storage devices is displayed on this screen. Here, the GPIO pins of Raspberry Pi come in handy. This allows the user to select the necessary files that need to be transferred. All the necessary options are displayed on this TFT screen.

One of the new features of the proposed system is portability i.e. the ability of the device to be taken anywhere without any compromise. Portability can be accomplished by using a portable power bank ( 5 Volts and $3000 \mathrm{~mA}$ or more) to power up the device sufficiently. Hence, it will allow the user to transfer data among distinct storage devices anywhere.

\subsection{ARM Processor}

Advanced RISC machine (ARM) is a family of Reduced Instruction Set Computing (RISC) architectures which are used in computer processors [8]. The ARM processor is one of the most important components of the proposed device. It is most suited for portable devices as its robustness and interface choices are higher as compared to its contemporaries. It is responsible for handling input for the system. For every touch to the host controller which controls the touch screen, it sends a corresponding signal in order keep the screen updated and send commands to the File System Controller Module for processing [3].

\subsection{USB}

The Universal Serial Bus (USB) is among the most convenient and frequently used data transmission interfaces currently. It has also been availed as an accepted configuration for the Personal Computer (PC) [6].

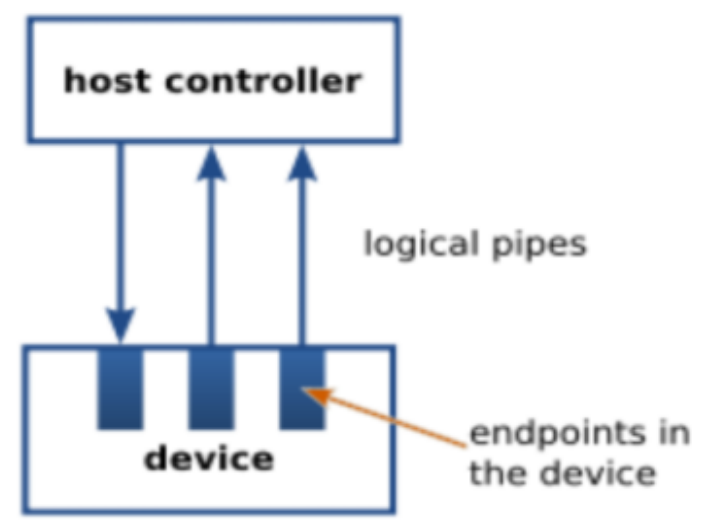

Fig. 2. USB pipes 
USB device communication is based on pipes. Pipes are nothing but logical channels. It is a connection from the host controller to a logical entity, found on a host device, and named an end point. USB devices can possess up to 32 endpoints (16 IN, 16 OUT) [10].

Two types of pipes are there: stream and message.

A message pipe is bi-directional pipe. It is used for control transfers. Message pipes are usually utilized for giving short and simple commands to the device.

A stream pipe is a unidirectional pipe which connected to a uni-directional endpoint. A stream pipe transfers data using isochronous, interrupt or bulk transfer [11].

Isochronous transfers: Isochronous transfer is performed for some fixed data rate. However, data loss may take place.

This type of data transfer is suitable for files between $20 \mathrm{~KB}$ and $1024 \mathrm{~KB}$.

Interrupt transfers: This type of transfer is used for the devices which need quick but guaranteed response. This type of data transfer takes place for files up to $20 \mathrm{~KB}$.

Bulk transfers: Bulk transfer utilizes the available bandwidth with no fixed data rate. This type of data transfer takes place for files more than $1024 \mathrm{~KB}$.

\subsection{Bluetooth Technology}

Bluetooth is a wireless communication standard used for exchanging data over short distances (using short-wavelength UHF radio waves in the ISM band) from mobile and fixed devices and building personal area networks (PANs). Bluetooth is a packet-based protocol with a master-slave structure. One master can communicate with up to seven slave elements. It uses a radio technology called FHSS (frequency hopping spread spectrum). The transmitted data is split up into packets and each data packet is transmitted on one of the 79 designated Bluetooth channels. Each channel has a bandwidth of $1 \mathrm{MHz}$. Bluetooth's physical range is typically less than $10 \mathrm{~m}$. Bluetooth module has been incorporated into the proposed system which allows wireless data transfer between devices with Bluetooth compatibly but no Wi-Fi compatibility [12].

\section{$4 \quad$ Detailed Working}

There have been provided various ways in which the user can transfer data from one storage device to another storage device. These are:

1. Data transfer between two USB storage devices.

2. Data transfer between a USB storage device and a Bluetooth compatible device.

Depending on the user's convenience, either a TFT LCD screen can be mounted on the GPIO pins of the microcontroller or, the content of the storage devices can be CASTED on a smart phone by using the mobile phone's IP address. 


\subsection{Casting}

Casting is a technology which allows a user to display his/her device's content on another screen using Wi-Fi as a medium.

When casting is initiated the host device starts a server to handle the exchange of screen casting data. It also connects to the Wi-Fi network to show its presence to all other devices in the same network.

After this, the host device identifies all the devices which are on the same Wi-Fi network and running a screen cast server on their system. This becomes possible by sending a message to every device which can be understood and replied by only a screen cast compatible display. They also help the device pair with its target display.

The cast screen connection is said to be initiated when both the device and the display get connected. The host device records its own screen and sound, encodes them into the H.264 video format and send to the display immediately at the same time as recording.

The target display simply plays this continuous streaming

\subsection{Data transfer between two USB storage devices.}

When the device is switched ON and the user connects the two USB storage devices (device A and device B), the USB host controller detects both the storage devices as it has the ability to detect the USB devices connected to its USB ports.

Once both the USB devices have been connected and detected, the user has to start the command prompt and enter the path of the USB devices i.e. two command prompt windows for two USB devices. In order to perform the various operations, dedicated keys have been provided.

1. Select the file(s) to perform the operations on.

2. If the file(s) need to be copied, go to 3, if the file(s) need to be moved, go to 4 , to delete the file(s), go to 5 , to rename a file, go to 6 , to compress a file, go to 7 and to uncompress a file, go to 8 .

3. Press F5 and there will be displayed a small window displaying the source as well as the destination directory.

3.1 Select the desired destination directory.

3.2 Select the option "yes" to start the transfer process.

3.3 If the transfer process needs to be aborted, select the "cancel" option present under the progress bar, else go to 1.

4. Press F6 and there will be displayed a small window displaying the source as well as the destination directory.

4.1 Select the desired destination directory.

4.2 Select the option "yes" to start the transfer process.

4.3 If the transfer process needs to be aborted, select the "cancel" option present under the progress bar, else go to 1.

5. Press F8 and delete the selected file(s). Then go to 1 . 
6. Press F2 and rename the selected file. Then go to 1.

7. Press F12 to display the menu on the screen. From the available options, select the "compress directory to" option and select the required extension in which the file needs to be compressed and go to 1 .

8. Press F12 to display the menu and then select the option uncompress. Then go to 1 .

\subsection{Data transfer between a USB storage device and a Bluetooth compatible device.}

There may arise certain situations when the user needs to share data among two non-compatible devices. More precisely, let's say transferring data from a Pen drive to a Bluetooth compatible device.

In such situation, the user uses the Bluetooth transfer application which has been given in the proposed device.

1. Open the application.

2. In order to search and pair with a new device, go to 3 and to transfer files to an already paired device. Then go to 4.1 .

3. Choose the "make discoverable" option to switch ON the Bluetooth of the

4. source storage device and search other Bluetooth compatible devices which have also been switched ON. Then go to 4 .

5. Select the "setup new device" option to pair with the desired destination storage device. Then go to 4.1 .

5.1 Browse and select the files to be transferred and then select the send option to dispatch the files. Then go to 2 .

\section{$5 \quad$ Results}

Following table shows the comparison between data transfer rate of a PC and the proposed system.

Files with different types of extensions and sizes have been chosen for comparison. It can be very well noted that if a PC takes 3 minutes to transfer a video file of size $902 \mathrm{mb}$ to a pen drive, the proposed system completes this transfer process within 98 seconds, which is less than even half of the time taken by the PC. Apart from a video file, several other files have also been transferred using both the systems. The time taken by a PC to transfer a text file is approximately $54 \mathrm{~ms}$ whereas the same file has been transferred by the proposed system in just $29 \mathrm{~ms}$.

A pdf document of size $318 \mathrm{~kb}$ got transferred in 1 second $82 \mathrm{~ms}$ by a PC whereas it took only $84 \mathrm{~ms}$ to transfer the same document by the proposed system. Music files are shared quite frequently nowadays. Hence an mp3 file of size $3 \mathrm{mb}$ has also been shared using both the systems.

To transfer the mp3 file, the PC took 2 seconds whereas the proposed system transferred the same file in just 1 second. 
Table 1. Data Transfer Seed using pc and the proposed system

\begin{tabular}{|l|c|c|}
\hline \multicolumn{1}{|c|}{ File Size } & Transfer time using PC & Transfer time using Proposed system \\
\hline Video file (902 MB) & $3 \mathrm{~min}$ & $1 \mathrm{~min} 38 \mathrm{sec}$ \\
\hline Text File $(82.6 \mathrm{~KB})$ & $54 \mathrm{~ms}$ & $29 \mathrm{~ms}$ \\
\hline PDF document $(318 \mathrm{~KB})$ & $1 \mathrm{sec} 82 \mathrm{~ms}$ & $84 \mathrm{~ms}$ \\
\hline Image $(331 \mathrm{~KB})$ & $1 \mathrm{sec} 63 \mathrm{~ms}$ & $60 \mathrm{~ms}$ \\
\hline Pptx $(264 \mathrm{~kb})$ & $92 \mathrm{~ms}$ & $44 \mathrm{~ms}$ \\
\hline Audio(3MB) & $2 \mathrm{sec}$ & $1 \mathrm{sec}$ \\
\hline
\end{tabular}

By observing table 1 we can conclude that the transfer rate of the proposed system is faster than that of a PC.

For the better realization of the equivalence of the data transfer speed of PC and the proposed system, time taken to transfer different size files of same extension (.mp4) has been compared.

Corresponding graph has been given in Figure 3.

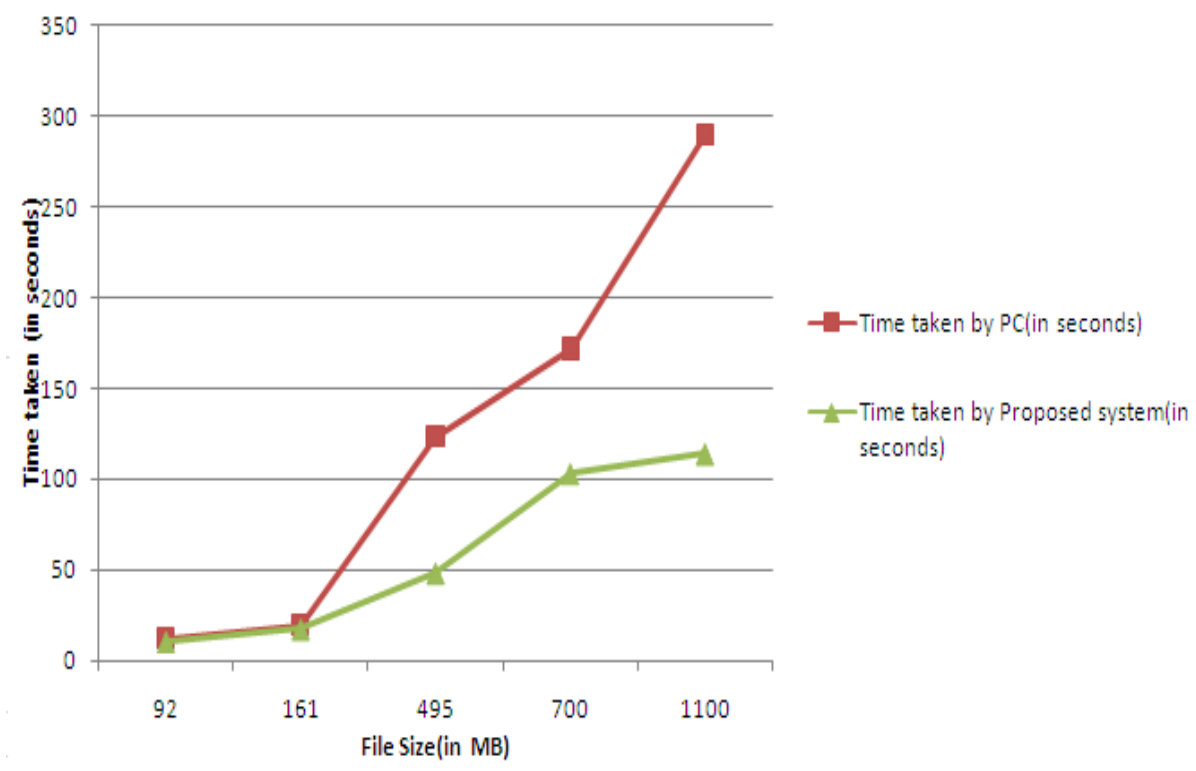

Fig. 3. Comparison between data transfer speed of $\quad \mathrm{PC}$ and proposed system for .mp4 files.

By considering the graph 1, it can be very well seen that even though for small size .mp4 files, time taken by both PC and the proposed system is almost same. But for large files, there is a huge difference between the performance of both the systems i.e. time taken by the proposed system is significantly less than the time taken by PC to transfer the same file. 


\section{Future Scope}

The proposed system in this paper can be further studied for some future scope. Since, the sole purpose of the system is to transfer data, it can't be used to read or open the files present in the storage devices. Also, the range for wireless data transfer can be improved by the help of Wi-Fi (WLAN) using various wireless communication protocols. However, it will require a deep and meticulous study of the proposed system. Hence, the future scope associated with the proposed system is that it can be studied by researchers and some research can be done to add the above mentioned functionalities.

\section{$7 \quad$ References}

[1] Sukhada M. Deshmukh, Prof. R. C. Mahajan, "Handheld Device for Data Transfer between Two USB Sticks", 2015 International Conference on Information Processing(ICIP).

[2] Chandrasinha D Parmar,V. V. Dwivedi,H. H. Wandra, "Firmware for File Transfer Between Bluetooth module Flash memory through microcontroller in an Emerging concept of Wireless Portable Memory Access", 2015 Communication Control and Computing Technologies (ICCCCT).

[3] Tushar Sawant, Bhagya Parekh, Naineel Shah "Computer Independent USB to USB Data Transfer Bridge", $20136^{\text {th }}$ International Conference on Emerging trends in Engineering and Technology. https://doi.org/10.1109/icetet.2013.8

[4] Khakal S. M.,Awari M.V., Gawner A.U., "USB Slave-to-Slave File Transfer Device", 2014,International Journal of Advances Electronics and Communication System.

[5] Mario A. Gomez-Rodriguez, Victor J. Sosa-Sosa and Ivan Lopez-Arevalo, "Adaptive File Transfer Middleware for mobile Applications", Data Communication Networking (DCNET), Proceedings of the 2010 International Conference.

[6] Zhnag Xiaoyan, Tie Yong, Li Dong "Design and Realization of an embedded storage system based on LPC2387 microprocessor", 2010 International Conference on Computer Application and System Modelling (ICCASM 2010).

[7] https://www.raspberrypi.org/products/raspberry-pi-2-model-b/

[8] Wikipedia https://en.m.wikipedia.org/wiki/ARM_architecture

[9] J. Ducloux, P. Petrashin, W. Lancioni, L. Toledo "An Embedded USB Dual-Role System Integrated for Mobile Devices", 2012 Argentine School of Micro-Nanoelectronics, Technology and Applications (EAMTA).

[10] Wikipedia https://en.m.wikipedia.org/wiki/USB_flash_drive

[11] Mr. TusharSawant, Mr. Sanjay Deshmukh, "USB to USB Data Transfer without PC",National Conference on Research in Electronics Advances Collaborative Technologies, 2013.

[12] Wikipedia https://en.m.wikipedia.org/wiki/Bluetooth

\section{Authors}

Darshana Rarath is a final year computer engineering student pursuing her degree from Dr. D Y Patil School of engineering and technology, Pune, India which is affiliated to Savitribai Phule Pune University. 
Mayank Sharma is a final year computer engineering student pursuing his degree from Dr. D Y Patil School of engineering and technology, Pune, India which is affiliated to Savitribai Phule Pune University.

Akshay Mane is a final year computer engineering student pursuing his degree from Dr. D Y Patil School of engineering and technology, Pune, India which is affiliated to Savitribai Phule Pune University.

Pooja Dabral is a final year computer engineering student pursuing her degree from Dr. D Y Patil School of engineering and technology which is affiliated to Savitribai Phule Pune University.

Dr. Roshani Ade is a research scholar and Dean at Dr. D Y Patil School of engineering and technology, Pune, India. Her main research interest include machine learning and data mining.

Article submitted 23 April 2017. Published as resubmitted by the authors 27 May 2017. 